\title{
On the Number of Arrangements of Pseudolines
}

\author{
Stefan Felsner \\ Freie Universität Berlin, \\ Fachbereich Mathematik und Informatik, \\ Takustr. 9, 14195 Berlin, Germany \\ E-mail: felsner@inf.fu-berlin.de
}

\begin{abstract}
Given a simple arrangement of $n$ pseudolines in the Euclidean plane, associate with line $i$ the list $\sigma_{i}$ of the lines crossing $i$ in the order of the crossings on line $i$. $\sigma_{i}=\left(\sigma_{1}^{i}, \sigma_{2}^{i}, . ., \sigma_{n-1}^{i}\right)$ is a permutation of $\{1, . ., n\}-\{i\}$. The vector $\left(\sigma_{1}, \sigma_{2}, \ldots, \sigma_{n}\right)$ is an encoding for the arrangement. Define $\tau_{j}^{i}=1$ if $\sigma_{j}^{i}>i$ and $\tau_{j}^{i}=0$, otherwise. Let $\tau_{i}=\left(\tau_{1}^{i}, \tau_{2}^{i}, . ., \tau_{n-1}^{i}\right)$, we show that the vector $\left(\tau_{1}, \tau_{2}, \ldots, \tau_{n}\right)$ is already an encoding.

We use this encoding to improve the upper bound on the number of arrangements of $n$ pseudolines to $2^{0.6988 \cdot n^{2}}$. Moreover, we have enumerated arrangements with 10 pseudolines. As a by-product we determine their exact number and we can show that the maximal number of halving lines of 10 point in the plane is 13 .
\end{abstract}

\section{Introduction}

Arrangements of lines and pseudolines are recognized as important and appealing objects for research in geometry and combinatorics. A general theory of arrangements is given in Grünbaum's monograph [8]. The oriented matroid point of view on arrangements is taken in Björner et al. [2]. Enumeration questions for arrangements are discussed in ([2], Subsection 6.5) and in Knuth ([9], Section 9). In most texts arrangements of pseudolines are defined with the real projective plane as ambient space. In contrast, we consider arrangements in the Euclidean plane.

Let a pseudoline be an $x$-monotone curve in the Euclidean plane. An arrangement of pseudolines is a family of pseudolines with the property that each pair of pseudolines has a unique point of intersection where the two pseudolines cross. An arrangement is simple if no three pseudolines have a common point of intersection. Throughout this manuscript the term arrangement if not specified further will always denote a simple arrangement of pseudolines. The size of an arrangement is the number of its pseudolines. Given an arrangement $\mathcal{A}$ of size $n$ we label the pseudolines so that they cross a vertical line left of all intersections in increasing order from bottom to top.

An arrangement partitions the plane into cells of dimensions 0,1 or 2 , the vertices, edges and faces of the arrangement. The cells of an arrangement carry a natural lattice structure. Adding a $\mathbf{0}$ and a $\mathbf{1}$ element we obtain the face lattice of the arrangement. Two arrangements are considered to be isomorphic if their face lattices are isomorphic under the correspondence induced by their labelings.

Particularly nice pictures of arrangements of pseudolines are given by their wiring diagrams introduced in Goodman [5], see Figure 1. Let $\mathcal{W}$ be a wiring diagram of a simple 
arrangement of size $n$. For each abscissa $x$ where no crossing takes place the vertical order (upwards) of the pseudolines at $x$ is a permutation $\pi_{x}$ of $\{1 . . n\}$. Assuming that no two crossings of $\mathcal{W}$ have the same $x$ position we obtain $\left(\begin{array}{l}n \\ 2\end{array}\right)+1$ different permutations. Denote by $\Sigma$ the sequence of these permutations in left to right order. We note two properties of sequence $\Sigma$.

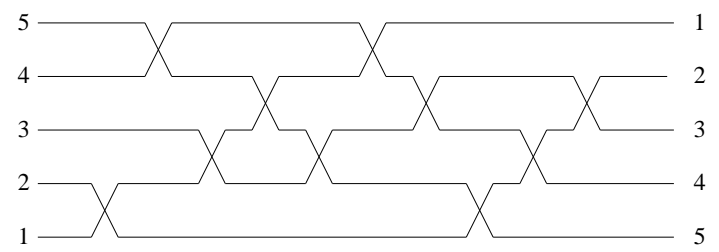

Figure 1. Wiring diagram.

(1) The first element of $\Sigma$ is the identity permutation $(1,2, \ldots, n)$ and the last element of $\Sigma$ is the reverse permutation $(n, \ldots, 2,1)$.

(2) Two consecutive permutations in $\Sigma$ differ by the reversal of an adjacent pair.

Following Goodman and Pollack [6,7] we call a sequence $\Sigma$ of $\left(\begin{array}{l}n \\ 2\end{array}\right)+1$ permutations of $\{1 . . n\}$ satisfying the above properties a simple allowable sequence. In general allowable sequences it is allowed for consecutive permutations to differ by the reversal of a larger substring. A simple allowable sequence is easily transformed into a wiring diagram and, hence, an arrangement of pseudolines. Note, however, that many allowable sequences may correspond to the same arrangement. Consecutive pairs of crossings that have no pseudoline in common can be interchanged without changing the arrangement.
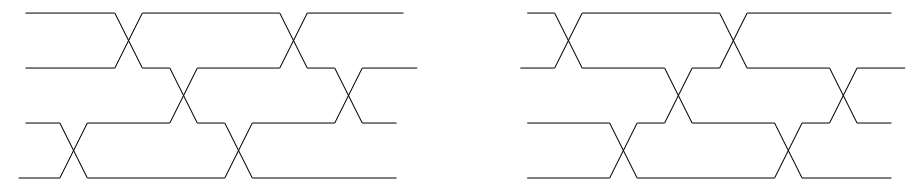

Figure 2. Wiring diagrams corresponding to one arrangement but two allowable sequences.

Simple allowable sequences are basically the same as reflection networks, see Knuth [9]. Alternatively, they can also be seen as maximal chains in the weak Bruhat order of the symmetric group. In this last context their number $A_{n}$ has been determined by Stanley [10]. His remarkable formula is

$$
A_{n}=\frac{\left(\begin{array}{l}
n \\
2
\end{array}\right) !}{\prod_{k=1}^{n-1}(2 n-2 k-1)^{k}} .
$$

Edelman and Greene [3] prove this formula via a combinatorial bijection between different types of tableaux.

Let $B_{n}$ be the number of non-isomorphic simple arrangements of size $n$. Besides the numbers $A_{n}$ and $B_{n}$ we will consider their logarithms $a_{n}=\log _{2} A_{n}$ and $b_{n}=\log _{2} B_{n}$. From the above remarks it follows that there are more allowable sequences than arrangements, 
i.e., $b_{n}<a_{n}$. From Stanley's formula an $O\left(n^{2} \log n\right)$ upper bound for $a_{n}$ follows. Knuth [9] proves lower and upper bounds for the number of arrangements:

$$
2^{\frac{n^{2}}{6}-\frac{5 n}{2}} \leq B_{n} \leq 3^{\left(\begin{array}{c}
n+1 \\
2
\end{array}\right)}
$$

This gives $b_{n} \leq 0.7924\left(n^{2}+n\right)$. Knuth reports on some computations supporting a conjecture of $b_{n} \leq\left(\begin{array}{c}n \\ 2\end{array}\right)$. From the sharpest version of the zone theorem (Bern et al. [1]) a bound of $b_{n} \leq 0.7194 n^{2}$ is obtained. In the next section we propose a new encoding of arrangements from which we easily obtain $b_{n} \leq 0.7213 n^{2}$. In Section 3 we work a little harder to obtain an improved bound of $b_{n} \leq 0.6988 n^{2}$.

\section{An encoding for arrangements}

Representing an arrangement by an allowable sequence can be seen as an encoding by an ordered sequence of vertical cuts through the arrangement. We turn the picture and give a representation by a sequence of horizontal cuts. An obvious way to do this is to associate with line $i$ the list $\sigma_{i}$ of the lines crossing $i$ in the order of the crossings on line $i$. To an arrangement $\mathcal{A}$ thus corresponds a vector $\left(\sigma_{1}, \ldots, \sigma_{n}\right)$ where $\sigma_{i}$ is a permutation of $\{1, . ., i-1, i+1, . ., n\}$. As will be shown in this section it suffices to know which entries of $\sigma_{i}$ are larger than $i$ in order to obtain an encoding for $\mathcal{A}$.

Definition 1 Let $\mathcal{T}_{n}$ be the set of $n$-tuples $\left(\tau_{1}, \tau_{2}, \ldots, \tau_{n}\right)$ with $\tau_{i}=\left(t_{1}^{i}, t_{2}^{i}, \ldots, t_{n-1}^{i}\right)$ a binary vector and $\sum_{j=1}^{n-1} t_{j}^{i}=n-i$ for all $i$.

Define a mapping $\Phi$ from arrangements of size $n$ to $\mathcal{T}_{n}$. Given an arrangement $\mathcal{A}$ let $\tau_{i}$ report the crossings of pseudoline $i$ with the other lines from left to right. More precisely $t_{j}^{i}=1$ if the $j$-th crossing on line $i$ is a crossing with a line with index larger than $i$. In the wiring diagram this corresponds to a move of wire $i$ up into the next track. Conversely $t_{j}^{i}=0$ if line $i$ is moving down at the $j$-th crossing, i.e., if the $j$-th crossing on line $i$ is a crossing with a line with index smaller than $i$. Each of the $n-1$ lines different from $i$ contributes exactly one crossing on line $i$ and $n-i$ of these lines have a larger label than $i$. This proves that $\left(\tau_{1}, \tau_{2}, \ldots, \tau_{n}\right)=\Phi(\mathcal{A})$ is in $\mathcal{T}_{n}$. E.g., the element of $\mathcal{T}_{4}$ corresponding to the arrangement represented by the wiring diagram of Figure 1 is

$$
T=((1,1,1,1),(0,1,1,1),(0,1,1,0),(1,0,0,0),(0,0,0,0)) .
$$

Of course, not all elements of $\mathcal{T}_{n}$ correspond to an arrangement, e.g., for $n=4$ we have 9 elements in $\mathcal{T}_{4}$ but only 8 arrangements. The element of $\mathcal{T}_{4}$ not in the image of $\Phi$ is $T=((1,1,1),(1,0,1),(0,1,0),(0,0,0))$.

Theorem 1 The mapping $\Phi$ is injective.

Proof. Algorithmically the tool of choice for the construction of the face lattice of an arrangement of pseudolines is a topological sweep (see Edelsbrunner and Guibas [4]). Below we make use of the sweep paradigm to prove that we can reconstruct $\mathcal{A}$ from $\Phi(\mathcal{A})$ and thus prove the theorem.

Imagine a sweep as a move of a topological line continuously from left to right across the plane. All incidences between cells of the arrangement are met by the line during this 
move. We discretize the line and replace it by a cut of edges of the arrangement. This is a list $\left(e_{1}, e_{2}, \ldots, e_{n}\right)$ of edges such that

(1) Edge $e_{1}$ is on the boundary of the bottom face, i.e., on the face containing the vertical ray to $-\infty$ and edge $e_{n}$ is on the boundary of the top face, i.e., the face containing the vertical ray to $+\infty$.

(2) For each $1 \leq i \leq n-1$ there is a face $F_{i}$ of the arrangement with edges $e_{i}$ and $e_{i+1}$ on its boundary.

To get from the bottom face to the top face every pseudoline has to be crossed. Since a cut consists of $n$ edges only it follows that the order of edges of a cut represents a permutation of the lines of the arrangement. The sweep begins at the leftmost cut consisting of all left unbounded edges. The permutation corresponding to this cut is the identity permutation.

An advance move corresponds to shifting the topological line cross a point of the arrangement. The admissible points for advance moves are those with both left edges in the current cut (Figure 3).
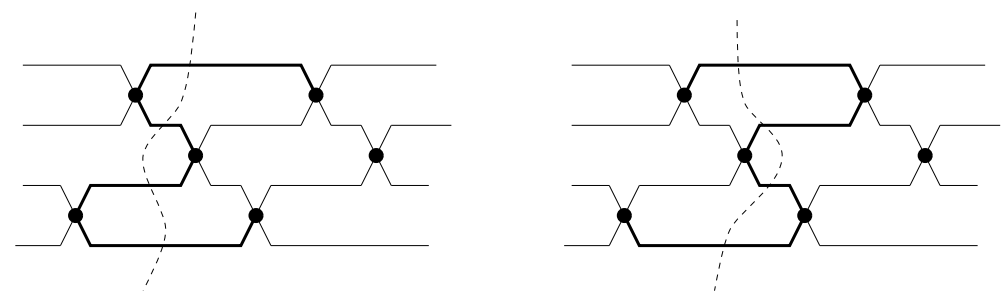

Figure 3. Advancing the cut across a vertex.

To have a deterministic algorithm our sweep always picks the highest admissible point for an advance move. Formally, let $i$ be the least index such that the right endpoints of edges $e_{i}$ and $e_{i+1}$ coincide in the current cut $\left(e_{1}, \ldots, e_{n}\right)$. The next cut is $\left(e_{1}, . ., e_{i-1}, e_{i}^{\prime}, e_{i+1}^{\prime}, e_{i+2}, . ., e_{n}\right)$ where $e_{i}^{\prime}$ is the edge right of $e_{i+1}$ on the same pseudoline and $e_{i+1}^{\prime}$ is the edge right of $e_{i}$ on the same pseudoline. In general, if two cuts differ by an advance move the corresponding permutations differ by an adjacent transposition. As long as some edges in the cut have right endpoints an advance move is possible. The algorithm terminates when the current cut has become the rightmost cut consisting of all right unbounded edges and the vertical order of the lines is reversed.

The next algorithm works with input $\Phi(\mathcal{A})$ and produces a sequence of permutations. The first permutation $\pi=\left(\pi_{1}, . ., \pi_{n}\right)$ is the identity. We initialize an edge counter $s(i)=1$ for each line $i$ and let $v_{i}=t_{s\left(\pi_{i}\right)}^{\pi_{i}}$ and $v=\left(v_{1}, v_{2}, \ldots, v_{n}\right)$. It will be important to keep in mind that $v$ depends on $\pi$ and $s$. Initially $v_{i}$ is simply the first bit of $\tau_{i}$ where $\Phi(\mathcal{A})=$ $\left(\tau_{1}, \ldots, \tau_{n}\right)$.

In each step the algorithm takes the least index $i$ with $v_{i}=1$ and $v_{i+1}=0$. Edge counters $s\left(\pi_{i}\right)$ and $s\left(\pi_{i+1}\right)$ are increased by one and $\pi$ is changed by an adjacent transposition at position $i$, i.e., $\pi$ becomes $\left(\pi_{1}, . ., \pi_{i-1}, \pi_{i+1}, \pi_{i}, \pi_{i+2}, . ., \pi_{n}\right)$.

The claim is that sweeping $\mathcal{A}$ and $\Phi(\mathcal{A})$ produces the same sequence of indices $i$ for advance moves. The following invariant suffices to prove the claim by induction.

$(\star) \quad v$ contains the information which lines go up and which go down at the next crossing behind the current cut in the order in which the lines appear in the cut. 
This is trivially verified at the beginning. Now suppose that $(\star)$ is true after some fixed number of moves of both algorithms.

If the next advance move of the sweep is made at index $i$ then $v_{i}=1$ and $v_{i+1}=0$ by induction and $i$ is a candidate index for the choice of $i$ in the algorithm with input $\Phi(\mathcal{A})$. Now let $j$ be any index with $v_{j}=1$ and $v_{j+1}=0$. This means that at its next crossing line $\pi_{j}$ is moving up while line $\pi_{j+1}$ is moving down at its next crossing. Since line $\pi_{j}$ is below line $\pi_{j+1}$ and they border a common face in $\mathcal{A}$ they cross each other, i.e., edges $e_{j}$ and $e_{j+1}$ have a common right endpoint. Therefore, $j$ is a candidate index for an advance move. The candidate sets for both algorithms thus agree and both choose the same (least) candidate. After the move the new $\pi$ is the permutation corresponding to the new cut. Since we have also increased the counters for the two lines involved in the crossing invariant $(\star)$ is seen to hold.

It has been remarked that the sequence of cuts produced by the sweep corresponds to a sequence of permutations. By $(\star)$ this sequence of permutations is exacly the sequence produced by the second algorithm. This sequence is a simple allowable sequence and characterizes an arrangement. Evidently this arrangement is again $\mathcal{A}$. This completes the proof that $\mathcal{A}$ can be reconstructed from $\Phi(\mathcal{A})$.

We have seen that $\Phi$ is an injective mapping from arrangements of size $n$ to elements of $\mathcal{T}_{n}$. Counting elements of $\mathcal{T}_{n}$ is a trivial task, $\left|\mathcal{T}_{n}\right|=\left(\begin{array}{c}n-1 \\ 0\end{array}\right)\left(\begin{array}{c}n-1 \\ 1\end{array}\right)\left(\begin{array}{c}n-1 \\ 2\end{array}\right) \ldots\left(\begin{array}{c}n-1 \\ n-1\end{array}\right)$. Taking logarithms and using Stirling's formula we obtain.

Fact 1. $b_{n}<\sum_{k=1}^{n-1} k \log e=0.7213\left(n^{2}-n\right)$.

Compared to the best known bound $b_{n} \leq 0.7194 n^{2}$ this was surprisingly easy to obtain.

For a better understanding of the encoding $\Phi$ it would be interesting to have some tools to discriminate between members from $\mathcal{T}_{n}$ that are in the image of $\Phi$ and those that are not. At this time we have little more than the second algorithm from the above proof. We can take arbitrary elements $T \in \mathcal{T}_{n}$ as input to this algorithm. The two possible outcomes are.

(1) The algorithm gets stuck before $\left(\begin{array}{l}n \\ 2\end{array}\right)$ moves have been made, i.e., in the current vector $V$ there is no index $i$ with $v_{i}=1$ and $v_{i+1}=0$.

(2) $T$ indeed corresponds to an arrangement.

Other cases can be ruled out as follows. Suppose that $T$ can be swept and consider the sequence of permutations generated. Since line $i$ moved up $n-i$ times and down $i-1$ line $i$ ends up on wire $n-i+1$. This proves that we end up with the reverse permutation. Hence, the sequence is allowable and corresponds to an arrangement.

\section{A better bound for $b_{n}$}

Recall the element $T=((1,1,1),(1,0,1),(0,1,0),(0,0,0))$ of $\mathcal{T}_{4}$ not in the image of $\Phi$. Trying to sweep $T$ we get stuck after two moves. At the second move we already note that something goes wrong since the lines involved in the crossing of the first move cross back. Call an immediate backcross a situation where two lines cross twice in a row. Geometrically this corresponds to two edges with the same left and right endpoints. When sweeping 
$T \in \mathcal{T}_{n}$ we recognize an immediate backcross when the pair $\left(v_{i}, v_{i+1}\right)=(1,0)$ of the move is replaced by $\left(v_{i}^{\prime}, v_{i+1}^{\prime}\right)=(1,0)$, i.e., the vectors $v$ and $v^{\prime}$ before and after the move are identical.

Note that the sweep corresponding to $T \in \mathcal{T}_{n}$ is completely determined by the initial vector $v$ and a sequence of replace pairs $w_{1}, w_{2}, \ldots, w_{\left(\begin{array}{c}n \\ 2\end{array}\right)}$. If the $j$ th move of the sweep interchanges $\pi_{i}$ and $\pi_{i+1}$ we replace $\left(v_{i}, v_{i+1}\right)=(1,0)$ by the pair $w_{j}=\left(w_{j}^{1}, w_{j}^{2}\right)$. A sequence of replace pairs leads to an immediate backcross exactly if one of the pairs $w_{j}$ is $(1,0)$. The number of backcross free elements of $\mathcal{T}_{n}$ and, hence, the number of arrangements can thus be estimated from above by the number of initial vectors $v$ and the number of $(1,0)$ free sequences of replace pairs. For $v$ there are $\leq 2^{n}$ choices and for each pair $w_{j}$ there remain 3 choices, therefore:

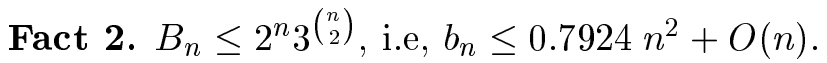

The proof of Fact 1 made use only of the number of 0 and 1 in each $\tau_{j}$. The proof of Fact 2 is based on forbidding immediate backcrossings. With the replace matrix we next define a representation that helps to take care of both aspects. Estimating the number of replace matrices will enable us to slightly improve the upper bound for $b_{n}$ in Theorem 2 .

Definition $2 A$ replace matrix is a binary $n \times n$ matrix $M$ with properties

$$
\begin{aligned}
& \sum_{j=1}^{n} m_{i j}=n-i \text { for } i=1, . ., n, \\
& m_{i j} \geq m_{j i} \text { for all } i<j .
\end{aligned}
$$

Lemma 1 There is an injective mapping $\Psi$ from arrangements of size $n$ to $n \times n$ replace matrices.

Proof. Consider $\Phi(\mathcal{A})$ and let $m_{i i}=t_{1}^{i}$, that is, we record the initial $v$ of the sweep of $\Phi(\mathcal{A})$ along the diagonal of $M$. If in the $k$ th move of the sweep of $\Phi(\mathcal{A})$ lines $i$ and $j$ cross we define $m_{i j}=1$ if the next crossing (after the crossing with line $j$ ) of line $i$ goes up and $m_{i j}=0$ if the next crossing of line $i$ goes down, respectivly $m_{i j}=t_{s(i)+1}^{i}$. If $i<j$ then at their crossing line $i$ is going up and line $j$ is going down. Since the lines don't backcross we have $\left(m_{i j}, m_{j i}\right) \neq(0,1)$ or equivalently $m_{i j} \geq m_{j i}$. After the complete sweep of $\Phi(\mathcal{A})$ we remain with a single undefined entry in each row of $M$. Let this entry be 0 . Suppose $i<j$ and $m_{i j}$ was the last undefined entry of its row. It follows that after crossing $j$ from below line $i$ was not involved in further crossings. If line $j$ had a further crossing then it had to move down there since the position above $j$ was occupied by $i$, hence, $m_{j i}=0$. Otherwise line $j$ had no further crossings and again $m_{j i}=0$.

Property (1) of replace matrices is easily seen to hold for $M$ as defined above. The entries in row $i$ of $M$ are the entries of $\tau_{i}$ in $\Phi(\mathcal{A})$ and an additional 0 in some permutation. Hence, $M=\Psi(\mathcal{A})$ is a well defined replace matrix. To show that this mapping is injective we sweep $M=\Psi(\mathcal{A})$ and reconstruct $\Phi(\mathcal{A})$. The details very similar to the arguments in the proof of Theorem 1 are left to the reader.

We illustrate this encoding of arrangements by replace matrices by giving the replace 
matrix corresponding to the arrangement of Figure 1. In that case

$$
M=\left(\begin{array}{lllll}
1 & 1 & 1 & 0 & 1 \\
1 & 0 & 0 & 1 & 1 \\
1 & 0 & 0 & 0 & 1 \\
0 & 0 & 0 & 1 & 0 \\
0 & 0 & 0 & 0 & 0
\end{array}\right)
$$

To obtain an estimate for the number of replace matrices we use probabilistic arguments. Consider the probability space $\Omega$ of all binary $n \times n$ matrices with $\sum_{j=1}^{n} m_{i j}=n-i$ for $i=1, . ., n$ and let $M$ be an uniformly distributed random variable in $\Omega$. Let $p_{i}$ be the probability that a fixed entry in row $i$ of $M$ is 0 , i.e., $p_{i}=\frac{i}{n}$, and $q_{i}=1-p_{i}$ be the probability that this entry is 1 , i.e., $q_{i}=\frac{n-i}{n}$.

For $i<j$ let $E_{i j}$ be the event $m_{i j} \geq m_{j i}$. Since $m_{i j} \nsucceq m_{j i}$ is equivalent to $\left(m_{i j}, m_{j i}\right)=$ $(0,1)$ the probability of event $E_{i j}$ is $\operatorname{Prob}\left[E_{i j}\right]=\left(1-p_{i} q_{j}\right)$. For the number $R_{n}$ of replace matrices we have $R_{n}=|\Omega| \operatorname{Prob}\left[\bigwedge_{i<j} E_{i j}\right]$.

Carelessly assuming independence of the events $E_{i j}$ we obtain as estimate for $R_{n}$ the product $\prod_{k=0}^{n-1}\left(\begin{array}{l}n \\ k\end{array}\right) \prod_{i<j}\left(1-\frac{i(n-j)}{n^{2}}\right)$. The logarithm of this function behaves like $0.66 n^{2}$. Of course due to the fixed row sums of matrices in $\Omega$ the $E_{i j}$ are not independent. In the remaining part of this section we derive a valid and weaker estimate for $R_{n}$.

Lemma 2 If $I$ is a subset of $\{(i, j): 1 \leq i<j \leq n-1\}$ such that $\operatorname{Prob}\left[E_{\alpha} \mid \wedge_{\beta \in J} E_{\beta}\right] \leq$ $\operatorname{Prob}\left[E_{\alpha}\right]$ for all $\alpha \in I$ and $J \subseteq I-\alpha$ then $R_{n} \leq|\Omega| \prod_{\alpha \in I} \operatorname{Prob}\left[E_{\alpha}\right]$.

Proof. For every enumeration $\alpha_{1}, . ., \alpha_{|I|}$ of $I$ we have $\operatorname{Prob}\left[\bigwedge_{i<j} E_{i j}\right] \leq \operatorname{Prob}\left[\bigwedge_{\alpha \in I} E_{\alpha}\right]=$ $\prod_{i=1}^{|I|} \operatorname{Prob}\left[E_{\alpha_{i}} \mid \bigwedge_{j<i} E_{\alpha_{j}}\right]$. The assumption on $I$ implies Prob $\left[E_{\alpha_{i}} \mid \bigwedge_{j<i} E_{\alpha_{j}}\right] \leq \operatorname{Prob}\left[E_{\alpha_{i}}\right]$ for all $i$.

Lemma 3 The set $I=\left\{(i, j): 1 \leq i \leq\left\lfloor\frac{n}{2}\right\rfloor<j \leq n\right\}$ obeys the condition of Lemma 2.

Proof. Let $\Omega(i, j)$ be the set of matrices that can be obtained from matrices of $\Omega$ by removing rows $i$ and $j$. Think of $\Omega(i, j)$ as the set of $(n-2) \times n$ matrices with rows indexed $1, . ., i-1, i+1, . ., j-1, j+1, . ., n$ and $\sum_{l=1}^{n} m_{k l}=n-k$ for index $k$. Given $M^{\prime} \in \Omega(i, j)$ let $\mathrm{N}\left(M^{\prime}\right)$ be the number of matrices $M$ in $\Omega$ that reduce to $M^{\prime}$ by removing rows $i$ and $j$, equivalently $\mathrm{N}\left(M^{\prime}\right)$ counts the number of pairs $\left(r_{i}, r_{j}\right)$ of rows that extend $M^{\prime}$ to a matrix in $\Omega$. Generalizing this notation let $\mathrm{N}\left(M^{\prime}: E\right)$ be the number of pairs of rows that extend $M^{\prime}$ to a matrix $M$ in $\Omega$ so that $E$ holds for $M$. Let $\alpha=(i, j) \in I$ and $J \subseteq I-\alpha$. The following inequalities are equivalent.

$$
\begin{aligned}
\operatorname{Prob}\left[E_{\alpha}\right] & \geq \operatorname{Prob}\left[E_{\alpha} \mid \bigwedge_{\beta \in J} E_{\beta}\right] \\
\operatorname{Prob}\left[\neg E_{\alpha}\right] & \leq \operatorname{Prob}\left[\neg E_{\alpha} \mid \bigwedge_{\beta \in J} E_{\beta}\right] \\
\operatorname{Prob}\left[\neg E_{\alpha}\right] \cdot \operatorname{Prob}\left[\bigwedge_{\beta \in J} E_{\beta}\right] & \leq \operatorname{Prob}\left[\neg E_{\alpha} \wedge \bigwedge_{\beta \in J} E_{\beta}\right] \\
\sum_{M^{\prime} \in \Omega(i, j)} \mathrm{N}\left(M^{\prime}: \neg E_{\alpha}\right) \sum_{M^{\prime} \in \Omega(i, j)} \mathrm{N}\left(M^{\prime}: \bigwedge_{\beta \in J} E_{\beta}\right) & \leq \sum_{M^{\prime} \in \Omega(i, j)} \mathrm{N}\left(M^{\prime}\right) \sum_{M^{\prime} \in \Omega(i, j)} \mathrm{N}\left(M^{\prime}: \neg E_{\alpha} \wedge \bigwedge_{\beta \in J} E_{\beta}\right) \\
\sum_{M^{\prime} N^{\prime} \in \Omega(i, j)} \mathrm{N}\left(M^{\prime}: \neg E_{\alpha}\right) \mathrm{N}\left(N^{\prime}: \bigwedge_{\beta \in J} E_{\beta}\right) & \leq \sum_{M^{\prime} N^{\prime} \in \Omega(i, j)} \mathrm{N}\left(M^{\prime}\right) \mathrm{N}\left(N^{\prime}: \neg E_{\alpha} \wedge \bigwedge_{\beta \in J} E_{\beta}\right)
\end{aligned}
$$


We claim that the last of these inequalities holds component-wise.

Claim. for any pair $M^{\prime}, N^{\prime}$ of matrices in $\Omega(i, j)$ :

$$
\mathrm{N}\left(M^{\prime}: \neg E_{\alpha}\right) \mathrm{N}\left(N^{\prime}: \bigwedge_{\beta \in J} E_{\beta}\right) \leq \mathrm{N}\left(M^{\prime}\right) \mathrm{N}\left(N^{\prime}: \neg E_{\alpha} \wedge \bigwedge_{\beta \in J} E_{\beta}\right)
$$

$\mathrm{N}\left(M^{\prime}\right)$ counts the number of pairs $\left(r_{i}, r_{j}\right)$ of row vectors that extend $M^{\prime} \in \Omega(i, j)$ to $M \in \Omega$. The condition on $r_{i}$ is $\sum_{l=1}^{n} r_{i l}=n-i$, there are $\left(\begin{array}{c}n \\ n-i\end{array}\right)$ choices for $r_{i}$. The number of choices for $r_{j}$ is $\left(\begin{array}{c}n \\ n-j\end{array}\right)$.

Now consider the pairs $\left(r_{i}, r_{j}\right)$ counted by $\mathrm{N}\left(M^{\prime}: \neg E_{\alpha}\right)$. To match condition $\neg E_{\alpha}$ the values $r_{i j}=0$ and $r_{j i}=1$ are required. There remain $\left(\begin{array}{l}n-1 \\ n-i\end{array}\right)$ choices for $r_{i}$ and $\left(\begin{array}{c}n-1 \\ n-j-1\end{array}\right)$ choices for $r_{j}$.

The number $\mathrm{N}\left(N^{\prime}: \bigwedge_{\beta \in J} E_{\beta}\right)$ really depends on $N^{\prime}$ respectively on the column vectors $s_{i}$ and $s_{j}$ of $N^{\prime}$. First consider the choices for $r_{i}$. To match the conditions $E_{\beta}$ for $\beta \in J$ certain relations between entries of $r_{i}$ and $s_{i}$ must hold. Note that due to the choice of $I$ we have $i \leq n / 2$ and all pairs containing $i$ in $J$ are of the form $(i, k)$, i.e., $n / 2<k$ and all relations forced between $s_{i}$ and $r_{i}$ are of the form $r_{i k} \geq s_{k i}$. Relevant for $r_{i}$ are only those positions with $s_{k i}=1$. Let $\lambda_{1}$ be the number of pairs $(i, k) \in J$ with $s_{k i}=1$, hence, conditions $E_{\beta}$ for $\beta \in J$ force exactly $\lambda_{1}$ positions $r_{i k}=1$. There remain $\left(\begin{array}{c}n-\lambda_{1} \\ n-i-\lambda_{1}\end{array}\right)$ choices for $r_{i}$. For $r_{j}$ note that all pairs containing $j$ in $J$ are of the form $(k, j)$, i.e., $k \leq n / 2<j$ and all relations forced between $s_{j}$ and $r_{j}$ are of the form $r_{k j} \leq s_{j k}$. Define $\lambda_{0}$ as the number of pairs $(k, j) \in J$ with $s_{j k}=0$. There remain $\left(\begin{array}{c}n-\lambda_{0} \\ n-j\end{array}\right)$ choices for $r_{j}$.

Finally, consider $\mathrm{N}\left(N^{\prime}: \neg E_{\alpha} \wedge \wedge_{\beta \in J} E_{\beta}\right)$. Compared to the previous case we have additionally fixed values $r_{i j}=0$ in $r_{i}$ and $r_{j i}=1$ in $r_{j}$. Hence, $\left(\begin{array}{c}n-\lambda_{1}-1 \\ n-i-\lambda_{1}\end{array}\right)$ choices for $r_{i}$ and $\left(\begin{array}{c}n-\lambda_{0}-1 \\ n-j-1\end{array}\right)$ choices for $r_{j}$. The claim is thus boiled down to the verification of

$$
\left(\begin{array}{c}
n-1 \\
n-i
\end{array}\right)\left(\begin{array}{c}
n-1 \\
n-j-1
\end{array}\right)\left(\begin{array}{c}
n-\lambda_{1} \\
n-i-\lambda_{1}
\end{array}\right)\left(\begin{array}{c}
n-\lambda_{0} \\
n-j
\end{array}\right) \leq\left(\begin{array}{c}
n \\
n-i
\end{array}\right)\left(\begin{array}{c}
n \\
n-j
\end{array}\right)\left(\begin{array}{c}
n-\lambda_{1}-1 \\
n-i-\lambda_{1}
\end{array}\right)\left(\begin{array}{c}
n-\lambda_{0}-1 \\
n-j-1
\end{array}\right) .
$$

Both of the following inequalities hold separately. Use $\left(\begin{array}{l}n \\ k\end{array}\right)=\frac{n}{n-k}\left(\begin{array}{c}n-1 \\ k\end{array}\right)$ and $\left(\begin{array}{l}n \\ k\end{array}\right)=$ $\frac{n}{k}\left(\begin{array}{c}n-1 \\ k-1\end{array}\right)$ for their proofs.

$$
\begin{aligned}
& \left(\begin{array}{c}
n-1 \\
n-i
\end{array}\right)\left(\begin{array}{c}
n-\lambda_{1} \\
n-i-\lambda_{1}
\end{array}\right) \leq\left(\begin{array}{c}
n \\
n-i
\end{array}\right)\left(\begin{array}{c}
n-\lambda_{1}-1 \\
n-i-\lambda_{1}
\end{array}\right) \\
& \left(\begin{array}{c}
n-1 \\
n-j-1
\end{array}\right)\left(\begin{array}{c}
n-\lambda_{0} \\
n-j
\end{array}\right) \leq\left(\begin{array}{c}
n \\
n-j
\end{array}\right)\left(\begin{array}{c}
n-\lambda_{0}-1 \\
n-j-1
\end{array}\right)
\end{aligned}
$$

Theorem 2 The number $B_{n}$ of arrangements of $n$ pseudolines is at most

$$
\prod_{k=0}^{n-1}\left(\begin{array}{l}
n \\
k
\end{array}\right) \prod_{1 \leq i \leq \frac{n}{2}<j \leq n}\left(1-\frac{i(n-j)}{n^{2}}\right)
$$

and hence $b_{n} \leq 0.6988 n^{2}$.

Proof. The above lemmas allows to bound the number $R_{n}$ of $n \times n$ replace matrices by $|\Omega| \prod_{(i, j) \in I}\left(1-\frac{i(n-j)}{n^{2}}\right)$. Plugging in $|\Omega|=\prod_{k=0}^{n-1}\left(\begin{array}{l}n \\ k\end{array}\right)$ and the definition of $I$ bounds $R_{n}$ by the above formula. By Lemma 1 the bound holds true for the number of arrangements. The proof is completed with a simple calculation. Recall that $1-p \leq e^{-p}$ for $0 \leq p$. Using 
this in every factor of the product and taking logarithms we obtain $r_{n} \leq \log _{2}(e)\left(\left(\begin{array}{c}n+1 \\ 2\end{array}\right)-\right.$ $\left.\sum_{(i, j) \in I} \frac{i(n-j)}{n^{2}}\right)$. The sum equals $\left(1 / n^{2}\right)\left(\sum_{i, j \leq n / 2} i \cdot j\right)=\left(1 / n^{2}\right)\left(\begin{array}{c}n / 2+1 \\ 2\end{array}\right)^{2} \approx(1 / 64) n^{2}$, altogether $r_{n} \leq \log _{2}(e)(1 / 2-1 / 64) n^{2}=0.6988 n^{2}$.

\section{Conclusion}

$B_{10}=18,410,581,880$. This is an additional value for the table of Knuth ([9], page 35).

As a byproduct of the computation for counting arrangements with 10 pseudolines we also found that the maximum number $h_{10}$ of halving-lines a set of 10 points in the plane can have is 13 . This adds a new value to the list $h_{4}=3, h_{6}=6$ and $h_{8}=9$. Via the duality between non-vertical lines and points $(y=a x+b) \leftrightarrow(a, b)$ a halving line of point-set $P$ corresponds to a cell $c$ in the arrangement dual to $P$ such that a vertical line through $c$ crosses half of the lines above and the other half below $c$. We call the set of these cells the middle-level of the arrangement. Note that the leftmost and the rightmost cell of the middle-level of an arrangement correspond to the same halving line in the dual. For more on the size of middle-levels and the more general $k$-set problem see [11], [7] and the references therein.

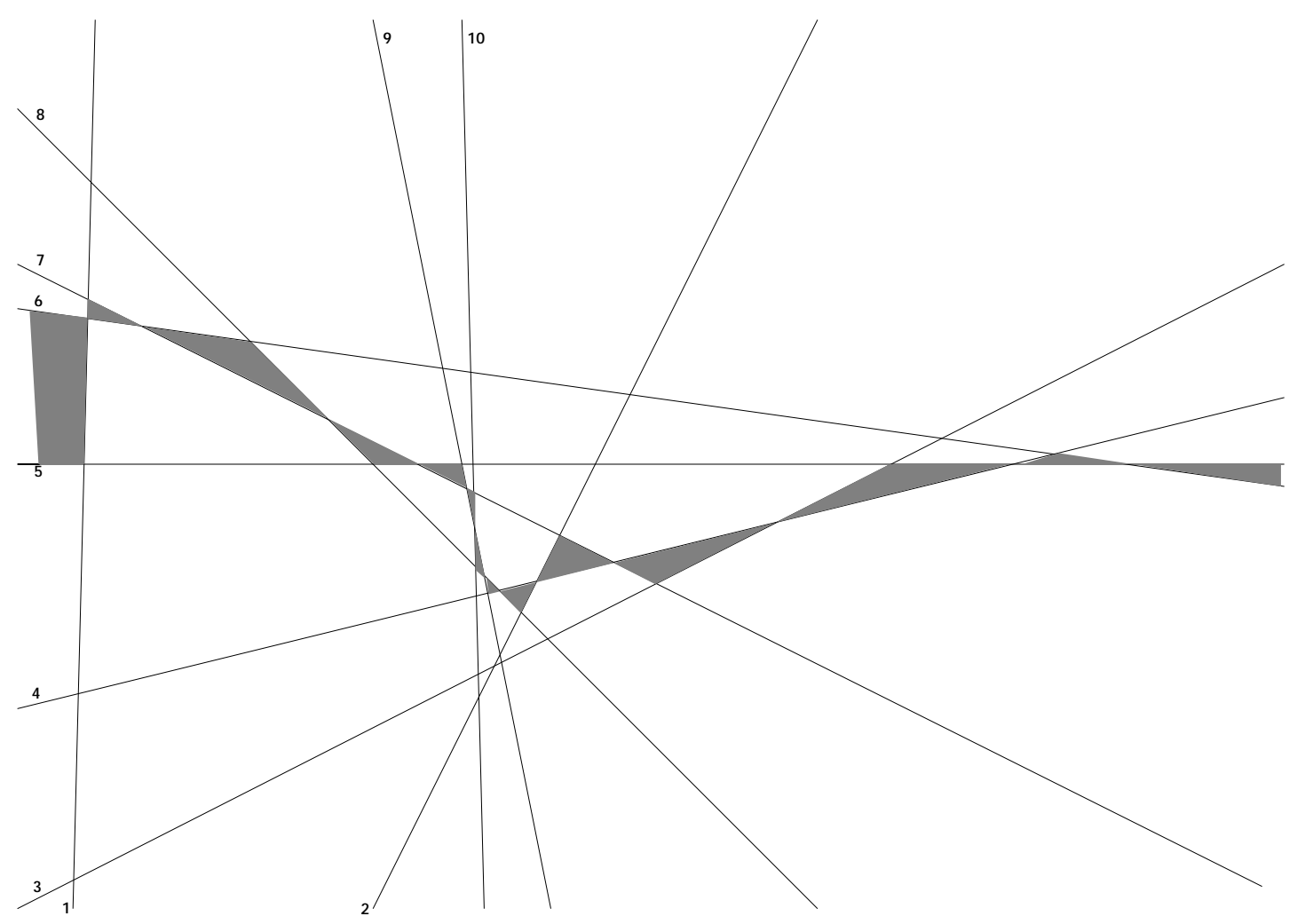

Figure 4. Ten lines with 14 cells in the middle-level.

\section{Acknowledgement.}

Thanks to Emo Welzl for support and encouragement. 


\section{References}

[1] M. Bern, D. Eppstein, P. Plassman, and F. Yao. Horizon theorems for lines and polygons. In J. Goodman, R. Pollack, and W. Steiger, editors, Discrete and Computational Geometry, pages 45-66. Amer. Math. Soc., 1991.

[2] A. Björner, M. Las Vergnas, B. Sturmfels, N. White, and G. Ziegler. Oriented Matroids. Cambridge University Press, Cambridge, 1993.

[3] P. Edelman and C. Greene. Balanced tableaux. Advances in Math., 63:42-99, 1987.

[4] H. Edelsbrunner and L. J. Guibas. Topologically sweeping an arrangement. J. Comput. Syst. Sci., 38:165-194, 1989. Corrigendum in 42 (1991), 249-251.

[5] J. E. Goodman. Proof of a conjecture of Burr, Grünbaum and Sloane. Discrete Math., 32:27-35, 1980.

[6] J. E. Goodman and R. Pollack. Semispaces of configurations, cell complexes of arrangements. J. Combin. Theory Ser. A, 37:257-293, 1984.

[7] J. E. Goodman and R. Pollack. Allowable sequences and order types in discrete and computational geometry. In J. Pach, editor, New Trends in Discrete and Computational Geometry, volume 10 of Algorithms and Combinatorics, pages 103-134. SpringerVerlag, 1993.

[8] B. Grünbaum. Arrangements and spreads. Regional Conf. Ser. Math., Amer. Math. Soc., number 10, Providence, RI, 1972.

[9] Donald E. Knuth. Axioms and Hulls, volume 606 of Lecture Notes in Computer Science. Springer-Verlag, Heidelberg, Germany, 1992.

[10] R. Stanley. On the number of reduced decompositions of elements of Coxeter groups. Europ. J. Combinatorics, 5:359-372, 1984.

[11] E. Welzl. More on $k$-Sets of Finite Sets in the Plane. Discrete Comput. Geom., 1:95-100, 1986. 